\title{
GB code: A grain boundary generation code
}

DOI: $10.21105 /$ joss. 00900

\section{Software}

- Review ct

- Repository ¿

- Archive ct

Submitted: 26 July 2018

Published: 23 September 2018

\section{License}

Authors of papers retain copyright and release the work under a Creative Commons Attribution 4.0 International License (CC-BY).

\section{R. Hadian ${ }^{1}$, B. Grabowski ${ }^{1}$, and J. Neugebauer ${ }^{1}$}

1 Max-Planck-Institut fuer Eisenforschung, Duesseldorf, Germany

\section{Summary}

Grain boundaries (GBs) are crystalline borders between single crystals in materials microstructure. They play an important role in mechanical, chemical or electronic response of materials and are therefore essential to materials science and physics.

GBs are geometrical entities with a large parameter space that has been well formulated within a coincident site lattice (CSL) mathematical framework (Sutton \& Balluffi, 1996). One important computational advantage of the CSL formalism is that it enables the construction of GBs in a periodic setup for atomistic simulations. GB_code (Hadian, 2018) uses the CSL construction to generate GB atomic structures (currently for cubic materials) systematically. It provides input atomic structures for large-scale atomistic simulations with interatomic potentials (as implemented e.g. in LAMMPS (Plimpton, 1995)) or ab initio, density-functional-theory (DFT) simulations (as implemented e.g. in VASP (Kresse \& Furthmüller, 1996)). These atomistic codes can further calculate different properties of the GBs. In addition to providing the input structures, the csl_generator.py script and the attached Jupyter notebooks have extra functionality to show how the CSL properties can be used to locate, classify and categorize different GBs and to extract detailed information about them.

GB_code is designed to be a command line tool as it is documented in detail in the README file of the repository, but the modules can also be accessed separately for example via the attached Jupyter notebooks. The code consists of two main scripts, csl_generator.py and gb_generator.py, that should be used in this order to produce the final GB structures. The attached Jupyter notebooks in the Test directory, Usage_of_GB_code.ipynb and Dichromatic_pattern_CSL.ipynb, input the two scripts as modules. The former addresses the general usage of the code with some extra tips and functions to locate GBs of interest, the latter depicts how CSL properties such as the overlapping patterns and displacement shift complete (DSC) vectors can be extracted and visualized. In the notebooks, two examples of the usage of the GB_code in our previous publications (Hadian, Grabowski, Race, \& Neugebauer, 2016, Hadian, Grabowski, Finnis, \& Neugebauer (2018)) have been shown as well.

GB_code uses the analytical and mathematical formulations of the following works of Sutton \& Balluffi (1996), Bollmann (1982), Grimmer, Bollmann, \& Warrington (1974). Some functionality from the code by Wojdyr (2013) on CSL has been used in a modified form in the GB_code.

\section{Statement of need:}

GB_code is an interactive toolbox to learn about grain boundaries and it is versatile for running high-throughput calculations. The target audience is students/scientists of materials science and physics at any level of familiarity with the topic. Extensive use of the NumPy library in GB_code results in faster execution, especially when computing large structures. The user will be guided through in a step-by-step manner on how to 
find and create the GB of interest. The code has been designed to be simple to use and instructive with a special attention to GB plane orientation, which is often lacking in other grain boundary creation codes.

\section{Acknowledgements}

R. Hadian would like to thank professor Mike Finnis for fruitful discussions. Funding from the European Union's Horizon 2020 research and innovation programme (Grant agreement No. 639211) is also gratefully acknowledged.

\section{References}

Bollmann, W. (1982). Crystal lattices, interfaces, matrices: An extension of crystallography. W. Bollmann. Retrieved from https://books.google.de/books?id=oBt0QgAACAAJ

Grimmer, H., Bollmann, W., \& Warrington, D. H. (1974). Coincidence-site lattices and complete pattern-shift in cubic crystals. Acta Crystallographica Section A, 30(2), 197-207. doi:10.1107/S056773947400043X

Hadian, R. (2018).GitHub repository. https://github.com/oekosheri/GB_code; GitHub.

Hadian, R., Grabowski, B., Finnis, M. W., \& Neugebauer, J. (2018). Migration mechanisms of a faceted grain boundary. Physical Review Materials, 2(4). doi:10.1103/PhysRevMaterials.2.043601

Hadian, R., Grabowski, B., Race, C. P., \& Neugebauer, J. (2016). Atomistic migration mechanisms of atomically flat, stepped, and kinked grain boundaries. Physical Review B, 94(16). doi:10.1103/PhysRevB.94.165413

Kresse, G., \& Furthmüller, J. (1996). Efficient iterative schemes for ab initio totalenergy calculations using a plane-wave basis set. Phys. Rev. B, 54, 11169-11186. doi:10.1103/PhysRevB.54.11169

Plimpton, S. (1995). Fast parallel algorithms for short-range molecular dynamics. Journal of Computational Physics, 117(1), 1-19. doi:10.1006/jcph.1995.1039

Sutton, A., \& Balluffi, R. (1996). Interfaces in crystalline materials. Clarendon Press. Retrieved from https://books.google.de/books?id=DMafQgAACAAJ

Wojdyr, M. (2013). Gosam. GitHub repository. https://github.com/wojdyr/gosam/blob/ master/csl.py; GitHub. 